\title{
Machine learning classification methods in hyperspectral data processing for agricultural applications
}

\author{
Jonáš Hruška \\ University of Trás-os-Montes e Alto \\ Douro \\ Quinta de Prados, \\ 5001-801 Vila Real \\ +351259350 356 \\ jonash@utad.pt
}

\author{
Telmo Adão \\ INESC Technology and Science \\ and University of Trás-os-Montes e \\ Alto Douro \\ Quinta de Prados, \\ 5001-801 Vila Real \\ +351259350 356 \\ telmoadao@utad.pt
}

Pedro Marques

University of Trás-os-Montes e Alto

Douro

Quinta de Prados,

5001-801 Vila Real

+351259350356

pedro.marques@utad.pt

\section{António Sousa}

INESC Technology and Science

and University of Trás-os-Montes e

Alto Douro

Quinta de Prados,

5001-801 Vila Real

+351259350356

amrs@utad.pt
António Cunha

INESC Technology and Science

and University of Trás-os-Montes e

Alto Douro

Quinta de Prados,

5001-801 Vila Real

+351259350 356

acunha@utad.pt

Raul Morais

INESC Technology and Science and University of Trás-os-Montes e Alto Douro

Quinta de Prados,

5001-801 Vila Real

+351259350 356

rmorais@utad.pt

\author{
Luís Pádua \\ University of Trás-os-Montes e Alto \\ Douro \\ Quinta de Prados, \\ 5001-801 Vila Real \\ +351259350356 \\ luispadua@utad.pt
}

\begin{abstract}
In agricultural applications hyperspectral imaging is used in cases where differences in spectral reflectance of the examined objects are small. However, the large amount of data generated by hyperspectral sensors requires advance processing methods. Machine learning approaches may play an important role in this task. They are known for decades, but they need high volume of data to compute accurate results. Until recently, the availability of hyperspectral data was a big drawback. It was first used in satellites, later in manned aircrafts and data availability from those platforms was limited because of logistics complexity and high price. Nowadays, hyperspectral sensors are available for unmanned aerial vehicles, which enabled to reach a high volume of data, thus overcoming these issues. This way, the aim of this
\end{abstract}

Permission to make digital or hard copies of all or part of this work for personal or classroom use is granted without fee provided that copies are not made or distributed for profit or commercial advantage and that copies bear this notice and the full citation on the first page. Copyrights for components of this work owned by others than ACM must be honored. Abstracting with credit is permitted. To copy otherwise, or republish, to post on servers or to redistribute to lists, requires prior specific permission and/or a fee. Request permissions from Permissions@acm.org.

ICGDA '18, April 20-22, 2018, Prague, Czech Republic

(C) 2018 Association for Computing Machinery.

ACM ISBN 978-1-4503-6445-4/18/04 ..\$15.00

DOI: https://doi.org/10.1145/3220228.3220242 paper is to present the status of the usage of machine learning approaches in the hyperspectral data processing, with a focus on agriculture applications. Nevertheless, there are not many studies available applying machine learning approach to hyperspectral data for agricultural applications. This apparent limitation was in fact the inspiration for making this survey. Preliminary results using UAV-based data are presented, showing the suitability of machine learning techniques in remote sensed data.

\section{CCS Concepts}

- Information systems $\sim$ Geographic information systems $\bullet$ Information systems $\sim$ Data analytics • Information systems $\sim$ Process control systems

\section{Keywords}

Agriculture; deep learning; hyperspectral data; machine learning; remote sensing.

\section{INTRODUCTION}

Hyperspectral data processing has significantly changed during the last decades. This fact was caused mainly because of the developments in new advanced sensors and processing equipment. Classification is usually the main goal of the hyperspectral data thus, most efforts have been put into this task and different approaches have been tested [1-5]. Lately, machine learning (ML) and deep learning (DL) approaches are becoming popular. 
New processing approaches allowed new applications in various fields, as in agriculture, which is the main scope of this paper. Monitoring of agricultural activities faces special challenges that are not common to other economic sectors, thus the use of remote sensing (RS) is necessary [6]. RS techniques facilitate to gather information over large areas with high revisit frequency, which is needed because of the strong seasonal patterns related to the biological lifecycle of crops [7]. The concept of monitoring and managing the crop production based on acquired data analysis is called Precision Agriculture (PA) also known as Precision Farming (PF) [8].

ML approaches applied to RS data are nowadays mostly used to crop identification or land use/cover classification [9-11]. However, in agriculture there are cases, like disease, weeds, or pest's detection, where regular multispectral images cannot provide the needed level of detail, and thus hyperspectral imagery needs to be used. Nevertheless, using hyperspectral data for this purpose has brought some challenges, like high number of bands, and need for advanced classification methods [12]. The connections between the need of more advance processing algorithms for very high dimensional data are highlighted in [13]. As previously mentioned, the focus of this study is applying ML to hyperspectral data acquired by using RS approaches for agricultural applications, since it provides the possibility to identify effected crops over large areas without the need of touching the ground and this kind of information will help farmers to make their crop management decisions more effective and increase their productivity. ML along with HSI has the potential to change the way we look at agriculture nowadays., Especially when developments in processing and data accessibility, caused mainly due to the miniaturization of hyperspectral components allowed their implementation on Unmanned Aerial Vehicles (UAV) platforms, whose operability is more flexible and less expensive than traditional platforms used for carrying the hyperspectral sensor, needed for accurate results, has increased.

\section{HYPERSPECTRAL DATA}

Hyperspectral data are acquired by passive optical sensors. It uses a fact that surface can reflect, absorb and transmit electromagnetic solar radiation [14]. The Sun is the primary source of radiation in a passive RS system. The solar energy modified by the atmosphere that was reflected gets back to the sensor where is converted into digital form [15]. The captured reflectance of light may then be decomposed into hundreds or thousands of contiguous spectral wavelength bands, for every pixel in the image ranging from ultraviolet to infrared, providing more detailed signatures [16]. An example of spectral signatures of selected materials can be seen in Figure 1.

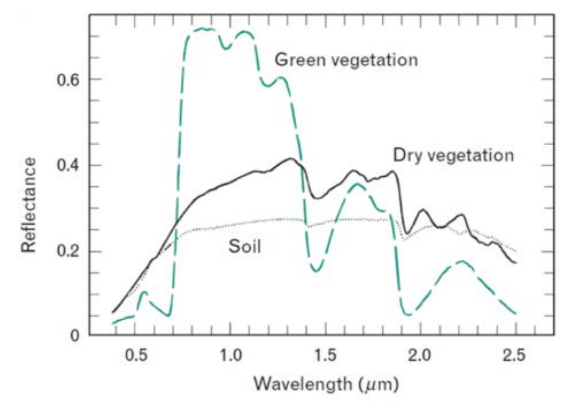

Figure 1. Spectral signatures of selected materials [17]
The signatures can be compared with spectral reflectance that were measured on materials with known compositions in laboratories or in the field, stored in spectral library. A combination of spectroscopy and image data acquisition by RS techniques, using different platforms (satellite, aircraft, UAV), is called hyperspectral imaging (HSI), known also as image spectroscopy. Each platform can be suitable for different applications depending on factors as coverage area or temporal resolution.

Hyperspectral images consist of hundreds or thousands of bands as opposed to multispectral images, that have usually from 5 to 12 bands [18]. The big number of spectral bands caused the emergence of third dimension to the spatial image resulting into 3D data cube. High dimensionality is special characteristics of hyperspectral images. In each band appears a unique brightness patterns or signatures of spectral bands. The rich spectral information available in the hundreds (eventually thousands) of narrow bands can possibly allow the accurate discrimination of different materials [3]. This fact makes hyperspectral data valuable. The biggest advantage of hyperspectral data, the high spectral dimensionality and resolution, is at the same time the biggest challenge to the processing and analyzing, arising the need for developing computationally efficient algorithms. In fact, standard parametric classifiers are not going to be sufficient anymore. ML appears to be a more suitable approach[19].

The problem with high dimensionality is known as "the curse of dimensionality", introduced by Bellman [20], named as the Hughes phenomenon [21-23]. It is usually present because of limited training samples regarding the size of the feature space [24], resulting in hardly recognizable patterns of curse dimensional feature spaces [25]. Limited number of training samples and high number of features can decrease the average accuracy [26]. Other effects can be seen as distance between neighboring samples which is almost uniform. If the dimension is increased towards infinity, the distance between maximum and minimum samples, when it is normalized, the minimum goes to zero (equation 1). That means that all samples are somehow distributed equally and as a consequence of that, almost all samples are close to some border of the space [20].

$$
\begin{gathered}
\lim _{n \rightarrow \infty} \frac{\text { dist }_{\max -} \text { dist }_{\min }}{\text { dist }_{\min }} \rightarrow 0 \\
\text { equation 1 Curse of dimensionality }
\end{gathered}
$$

To overcome the "curse of dimensionality" for hyperspectral data, the best technique for selecting the optimal dimensions needs to be chosen and preparation of sufficient training samples in the training phase of the classification needs to be done [26]. Principal Component Analysis (PCA), Linear Discriminant Analysis (LDA), Convex Cone Analysis (CCA), Independent Component Analysis (ICA), Nonnegative Matrix Factorization (NMF), Kernels and sparse reconstruction are the most known techniques for dimension reduction.

\section{MACHINE LEARNING}

$\mathrm{ML}$, as a part of artificial intelligence, allows to learn from input data and provides the ability to analyze complex data more effectively with higher classification accuracy than conventional processing methods [27], without being explicitly programmed [28]. The idea of applying ML into remote sensing was found in [29] back in 1990s. The ML approach in the study achieved highest accuracy compared to conventional methods at that time. After their great results and especially due to new advanced sensors that could produce data with higher quality in spatial and 
spectral resolution, needed for accurate outputs, and the availability of the data, ML has become an important tool in remote sensing analysis.

A number of ML algorithms has been presented to RS image analysis during last years, e.g. artificial neural networks (ANN), decision tree (DT) classifiers, Random Forest (RF) and Support Vector Machines (SVM) [30-32]. The SVM are in general context considered as a state of the art method for HSI data classification, because they handle the curse of dimensionality issue well, thus it can be applied to high dimensional data with limited dataset samples [33].

Lately, Deep Learning (DL) methods has proven to be very effective at discovering intricate structures in high-dimensional data [34], thus suitable for hyperspectral data. DL outperforms the limitation of conventional ML techniques, which was the ability to process natural data in their raw form [34]. The huge potential of DL hyperspectral data processing mentioned Mou et al. [35]. Zhang et al. [36] have found that DL is actually everywhere in remote sensing (RS) data analyzing (e. g. image preprocessing, pixel-based classification, target recognition and recently in highlevel semantic feature extraction and scene understanding), even though it did not provide the same impact on RS yet, as in detection in computer vision on RGB imagery [37].

DL is characterized by neural networks (NNs) with many hidden layers, that's why they are called deep [38]. Multiple hidden layers work to build an improved feature space. Higher layers make aspects of the input that are important for discrimination more relevant and suppress irrelevant variations. Second layer learns first order features, as edges; third layer learns higher order features (combination of first layer features, combinations of edges) [34].

Constructing a pattern-recognition or ML system required a deep knowledge for decades to build a feature extractor that could transformed the raw data into suitable internal representation or feature vector, from which the learning system could detect or classify patterns in input [34], but DL typically results in blackbox solutions that give us little to no insight into how they are working and why we should trust them [37]. DL is using representation learning. This approach has been known for decades but, due to recent advancement in computational power, more available data and excellent results, the implementation of the technique started to grow big. According to [38] it has even the fastest-growing trend in big data analysis, and it was consider as one of the ten breakthrough technologies of 2013 [39].

\section{APPLICATIONS}

\subsection{Agriculture applications}

Yalcin [40] utilized AlexNet as a pre-trained CNN model for classifying different phenological stages of six plant classes. The classification performance of the fine-tuned CNN based approach was compared with results achieved by ML approach based on manually extracted features. Results from manually extracted features were outperformed by results where the features were learned from CNN model. Liu et al. [41] applied Learning Vector Quantization (LVQ) neural network classifier and PCA techniques for discriminating and classifying different fungal infection levels in rice panicles. The classification accuracies achieved great results and proved that with hyperspectral remote sensing data it is possible to discriminate different fungal infection levels of rice panicles by using $\mathrm{NN}$ approach. A comparison of ML methods on hyperspectral plant disease assessments carried out Yeh et al. [42]. In the study were tested two hyperspectral image analysis methods: stepwise discriminant analysis (SDA), spectral angle mapper (SAM) and the proposed Simple Slope Measure (SSM) method. The outcomes of the research proved the suitability of using nondestructive methods to assess strawberry foliage Anthracnose symptoms in early stages. A comprehensive overview of ML tools for various biotic and abiotic stress traits applications can be found in [43]. Dutta et al. [44] provided a comparison of various supervised ML methods for a classification of salad leaf related disease. Fletcher and Turley [45] proved that by using combination of canopy hyperspectral narrowband data and random forest machine learning algorithm it is possible to differentiate cotton from Palmer amaranth, an invasive weed of cotton production systems. Ashourloo et al. [46] were applying ML regression techniques for the leaf rust disease detection using hyperspectral measurement. They found out that Gaussian process regression (GPR) can outperform other methods (partial least square regression, $v$ support vector regression) in case of using smaller training dataset and that challenges in early detection of plant disease can be reduced by ML regression techniques.

\subsection{Preliminary testing on vineyard detection}

With the goal to detect vineyard plots, a first approach using CNNs was carried out. Data acquisition from vineyards located within the campus of University of Trás-os-Montes and Alto Douro (UTAD) was carried out. Imagery was acquired using the fixed-wing UAV senseFly eBee (senseFly SA, Lausanne, Switzerland), with a Canon IXUS 127 HS camera - operating in RGB and a Canon PowerShot ELPH 110 HS camera - for rededge (RE) imagery acquisition, were used in two distinct flight plans. The flight height was $\sim 175 \mathrm{~m}$ resulting in $5 \mathrm{~cm} / \mathrm{px}$ Ground Sample Distance (GSD). Both RGB and RE imagery were submitted to a photogrametric processing stage that enabled orthophoto mosaics computation.

Afterwards, they were manually segmented in patches of $512 \mathrm{x}$ 512 pixels with stride of 64 pixels that were used to train a CNN (U-Net [47]). The network was implemented using Keras with TensorFlow as backend, coded in Python 2.7. All experiments were performed on workstation with an Intel Xeon CPU E5-2650, 64GB RAM and a NVIDIA GTX-1080Ti GPU (11 GB memory) which was used to accelerate the CNN. The CNN was trained from scratch with training set (Figure 2, left) which was split randomly in $80 \%$ (12.109 patches) for training and 20\% (3.002 patches) for testing. The training was made using Dice coefficient as cost function, in 3 cycles of 128 epochs with the Adam optimizer. The learning rate was $1 \mathrm{E}-4,1 \mathrm{E}-5$ and $1 \mathrm{E}-6$ in each cycle, respectively. Validation using Dice Coeficient achieved 0.9953 and is graphically depicted (Figure 2, right).

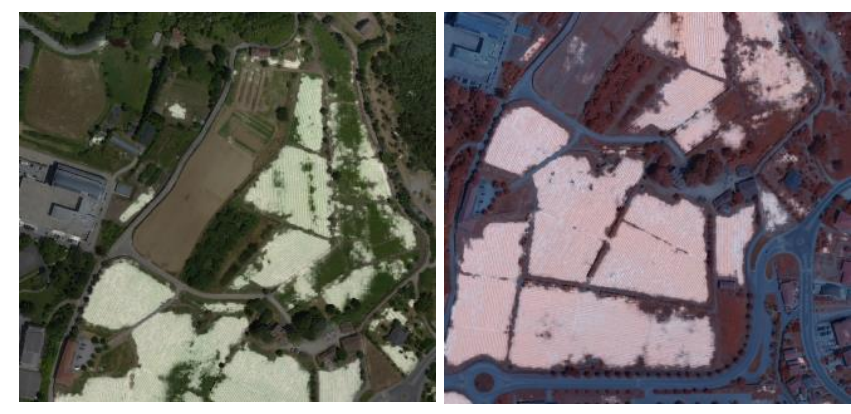

Figure 2. RGB (left) and RE (right) orthophoto mosaics of the vineyard used for training and testing with detected vineyard plots highlighted. 
The trained network was then applied to a different vineyard area for validation, with same parameters (patches and stride). The resulting image from classification was binarized by considering that all pixels with a value above zero correspond to vineyard plot. Then a set of morphological operations were applied to remove possible outliers and to provide a better understanding of the detected area. The morphological operations were applied in the following order: open, close, remove objects lower than 2000 pixels that correspond to potential outliers. For validation purposes, a pixelwise comparison with a manual segmented mask, which corresponds to ground truth, was performed. The result for exact detection was achieved with $85.07 \%$ accuracy, followed by $14.64 \%$ for under detection and $0.29 \%$ over detection. A visual interpretation of the obtained results is presented in Figure 3. It is noticeable a clear lack of detection in areas with strong shadow presence and less density of inter-row vegetation, being this nondetection related to the inexistence of such situations in the dataset used for learning.

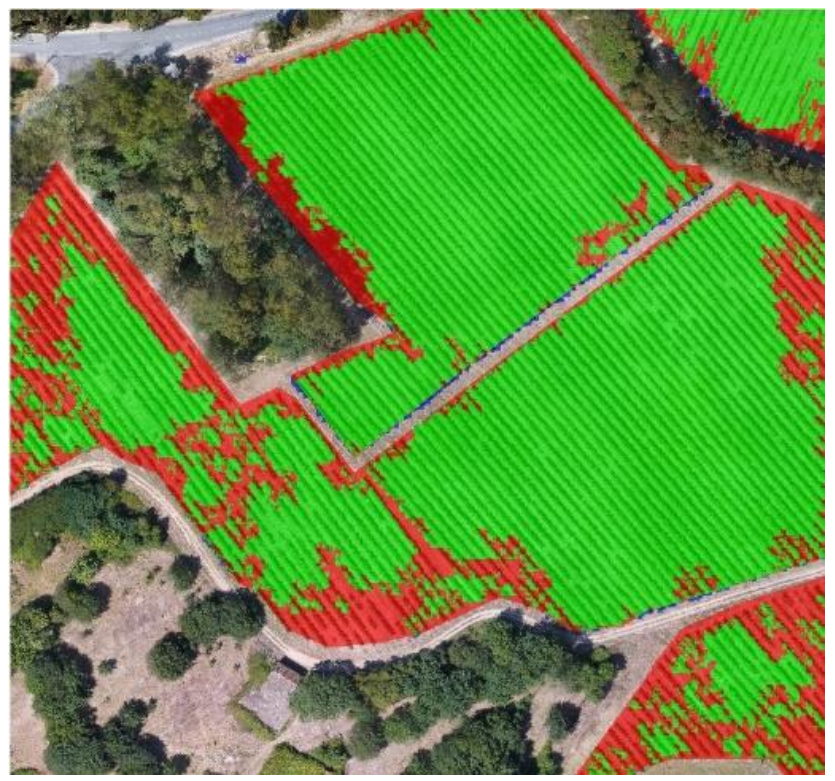

Figure 3. Results from the pixel-wise comparison of the detected vineyard plots against a manual segmented mask, with RGB orthophoto mosaic in the background. (red under detection, green exact detection and blue over detection).

\section{CONCLUSIONS AND FUTURE WORK}

Hyperspectral remote sensing imaging along with ML can bring some benefits to agricultural industry. It is a non-invasive, less laborious, accurate method that can reduce the costs and field work operations. Not many studies that implemented ML into remote sensing applications using hyperspectral data were found, especially in agriculture.

As for the obtained results, the good classification accuracy and experience acquired from these preliminary experiments encouraged the application of this approach for hyperspectral data processing in near future, driven by the strong conviction that ML/DL-based classification has a big potential for highdimensional data, which turns out to be justified by both literature and our experiments.

\section{ACKNOWLEDGMENTS}

This work was financed by the European Regional Development Fund (ERDF) through the Operational Programme for Competitiveness and Internationalisation - COMPETE 2020 under the PORTUGAL 2020 Partnership Agreement, and through the Portuguese National Innovation Agency (ANI) as a part of project "PARRA - Plataforma integrAda de monitoRização e avaliação da doença da flavescência douRada na vinhA" (No 3447) and ERDF and North 2020 - North Regional Operational Program, as part of project "INNOVINEandWINE - Vineyard and Wine Innovation Platform" (NORTE-01-0145-FEDER-000038).

\section{REFERENCES}

[1] Sabale, S.P. and Jadhav, C.R. 2015. Hyperspectral Image Classification Methods in Remote Sensing - A Review. 2015 International Conference on Computing Communication Control and Automation (Feb. 2015), 679683.

[2] Qiu, Q. et al. 2017. Survey of supervised classification techniques for hyperspectral images. Sensor Review. 37, 3 (Jun. 2017), 371-382.

[3] Ghamisi, P. et al. 2017. Advanced Spectral Classifiers for Hyperspectral Images: A review. IEEE Geoscience and Remote Sensing Magazine. 5, 1 (Mar. 2017), 8-32.

[4] Poojary, N. et al. 2015. Automatic target detection in hyperspectral image processing: A review of algorithms. 2015 12th International Conference on Fuzzy Systems and Knowledge Discovery (FSKD) (Aug. 2015), 1991-1996.

[5] Tarabalka, Y. 2010. Classification of hyperspectral data using spectral-spatial approaches. Institut National Polytechnique de Grenoble-INPG.

[6] Young, M.E. 2002. From early child development to human development: Investing in our children's future. World Bank Publications.

[7] Atzberger, C. 2013. Advances in Remote Sensing of Agriculture: Context Description, Existing Operational Monitoring Systems and Major Information Needs. Remote Sensing. 5, 2 (Feb. 2013), 949-981.

[8] Liaghat, S. et al. 2010. A Review: The Role of Remote Sensing in Precision Agriculture. American Journal of Agricultural and Biological Sciences. 5, 1 (Mar. 2010), $50-55$.

[9] Uba, N.K. 2016. Land use and land cover classification using deep learning techniques. Arizona State University.

[10] Elarab, M. 2015. The application of unmanned aerial vehicle to precision agriculture: Chlorophyll, nitrogen, and evapotranspiration estimation. Utah State University.

[11] Karalasa, K. et al. 2015. Deep learning for multi-label land cover classification. SPIE Remote Sensing (2015), 96430Q-96430Q.

[12] Prasad, S. et al. 2015. Introduction to the Issue on Advances in Hyperspectral Data Processing and Analysis. IEEE Journal of Selected Topics in Signal Processing. 9, 6 (Sep. 2015), 961-963.

[13] Benediktsson, J.A. et al. 2012. Very High-Resolution Remote Sensing: Challenges and Opportunities [Point of View]. Proceedings of the IEEE. 100, 6 (Jun. 2012), 19071910.

[14] Shippert, P. 2003. Introduction to hyperspectral image analysis. Online Journal of Space Communication. 3, (2003), 13.

[15] Manolakis, D. et al. 2003. Hyperspectral image processing for automatic target detection applications. Lincoln Laboratory Journal. 14, 1 (2003), 79-116.

[16] Yang, M. et al. 2015. Compressive hyperspectral imaging via adaptive sampling and dictionary learning. arXiv:1512.00901 [cs]. (Dec. 2015). 
[17] Geoinformatics|Digital Textbook Library: 2008. http://www.tankonyvtar.hu/en/tartalom/tamop425/0032_ter informatika/ch04s04.html. Accessed: 2018-02-06.

[18] Adão, T. et al. 2017. Hyperspectral Imaging: A Review on UAV-Based Sensors, Data Processing and Applications for Agriculture and Forestry. Remote Sensing. 9, 11 (Oct. 2017), 1110.

[19] Verleysen, M. et al. eds. 2013. Proceedings / 21st European Symposium on Artificial Neural Networks, Computational Intelligence and Machine Learning, ESANN 2013: Bruges, Belgium, April 24 - 25 - 26, 2013. Ciaco.

[20] Adaptive Control Processes: 1961. https://press.princeton.edu/titles/101.html. Accessed: 2018-01-14.

[21] Alajlan, N. et al. 2012. Fusion of supervised and unsupervised learning for improved classification of hyperspectral images. Information Sciences. 217, (Dec. 2012), 39-55.

[22] Alonso, M.C. et al. 2011. Consequences of the Hughes phenomenon on some classification techniques. Proceedings of the ASPRS 2001 annual conference (2011), $1-5$.

[23] Camps-Valls, G. et al. 2014. Advances in Hyperspectral Image Classification: Earth Monitoring with Statistical Learning Methods. IEEE Signal Processing Magazine. 31, 1 (Jan. 2014), 45-54.

[24] Plaza, A. et al. 2009. Recent advances in techniques for hyperspectral image processing. Remote Sensing of Environment. 113, (Sep. 2009), S110-S122.

[25] Gheyas, I.A. and Smith, L.S. 2010. Feature subset selection in large dimensionality domains. Pattern Recognition. 43, 1 (Jan. 2010), 5-13.

[26] Salimi, A. et al. 2017. Using a Feature Subset Selection method and Support Vector Machine to address curse of dimensionality and redundancy in Hyperion hyperspectral data classification. The Egyptian Journal of Remote Sensing and Space Science. (Mar. 2017).

[27] Shafri, H.Z.M. 2016. Machine Learning in Hyperspectral and Multispectral Remote Sensing Data Analysis. Artificial Intelligence Science and Technology. WORLD SCIENTIFIC. 3-9.

[28] Mitchell, T.M. 1997. Machine Learning. McGraw-Hill, Inc.

[29] Huang, X. and Jensen, J.R. 1997. A machine-learning approach to automated knowledge-base building for remote sensing image analysis with GIS data. Photogrammetric engineering and remote sensing. 63, 10 (1997), 1185-1193.

[30] Linden, S. van der et al. 2007. Classifying segmented hyperspectral data from a heterogeneous urban environment using support vector machines. Journal of Applied Remote Sensing. 1, 1 (Oct. 2007), 013543.

[31] Shang, X. 2013. Evaluating the capability of machinelearning algorithms and object-oriented classification techniques using hyperspectral remote sensing for the discrimination of Australian native forest species in southeastern Australia. University of Wollongong Thesis Collection 1954-2016. (Jan. 2013).

[32] Honkavaara, E. et al. 2012. HYPERSPECTRAL REFLECTANCE SIGNATURES AND POINT CLOUDS FOR PRECISION AGRICULTURE BY LIGHT WEIGHT UAV IMAGING SYSTEM. ISPRS Annals of
Photogrammetry, Remote Sensing and Spatial Information Sciences. I-7, (Jul. 2012), 353-358.

[33] Petersson, H. et al. 2016. Hyperspectral image analysis using deep learning \#x2014; A review. 2016 Sixth International Conference on Image Processing Theory, Tools and Applications (IPTA) (Dec. 2016), 1-6.

[34] LeCun, Y. et al. 2015. Deep learning. Nature. 521, 7553 (May 2015), 436.

[35] Mou, L. et al. 2017. Deep Recurrent Neural Networks for Hyperspectral Image Classification. IEEE Transactions on Geoscience and Remote Sensing. 55, 7 (Jul. 2017), 36393655.

[36] Zhang, L. et al. 2016. Deep Learning for Remote Sensing Data: A Technical Tutorial on the State of the Art. IEEE Geoscience and Remote Sensing Magazine. 4, 2 (Jun. 2016), 22-40.

[37] Ball, J.E. et al. 2018. Special Section Guest Editorial: Feature and Deep Learning in Remote Sensing Applications. Journal of Applied Remote Sensing. 11, 4 (Jan. 2018), 042601.

[38] Zhu, X.X. et al. 2017. Deep Learning in Remote Sensing: A Comprehensive Review and List of Resources. IEEE Geoscience and Remote Sensing Magazine. 5, 4 (Dec. 2017), 8-36.

[39] 10 Breakthrough Technologies 2013: 2013. https://www.technologyreview.com/lists/technologies/2013/. Accessed: 2018-01-15.

[40] Yalcin, H. 2017. Plant phenology recognition using deep learning: Deep-Pheno. 2017 6th International Conference on Agro-Geoinformatics (Aug. 2017), 1-5.

[41] Liu, Z.-Y. et al. 2010. Application of neural networks to discriminate fungal infection levels in rice panicles using hyperspectral reflectance and principal components analysis. Computers and Electronics in Agriculture. 72, 2 (Jul. 2010), 99-106.

[42] Yeh, Y.-H.F. et al. 2013. A Comparison of Machine Learning Methods on Hyperspectral Plant Disease Assessments. IFAC Proceedings Volumes. 46, 4 (2013), 361-365.

[43] Singh, A. et al. 2016. Machine Learning for HighThroughput Stress Phenotyping in Plants. Trends in Plant Science. 21, 2 (Feb. 2016), 110-124.

[44] Dutta, R. et al. 2015. Interactive visual big data analytics for large area farm biosecurity monitoring: i-EKbase system. Proceedings of the 4th International Conference on Big Data, Streams and Heterogeneous Source Mining: Algorithms, Systems, Programming Models and Applications-Volume 41 (2015), 9-18.

[45] Fletcher, R.S. and Turley, R.B. 2017. Employing Canopy Hyperspectral Narrowband Data and Random Forest Algorithm to Differentiate Palmer Amaranth from Colored Cotton. American Journal of Plant Sciences. 08, 12 (Nov. 2017), 3258.

[46] Ashourloo, D. et al. 2016. An Investigation Into Machine Learning Regression Techniques for the Leaf Rust Disease Detection Using Hyperspectral Measurement. IEEE Journal of Selected Topics in Applied Earth Observations and Remote Sensing. 9, 9 (Sep. 2016), 4344-4351.

[47] Ronneberger, O. et al. 2015. U-Net: Convolutional Networks for Biomedical Image Segmentation. arXiv:1505.04597 [cs]. (May 2015). 